\title{
The Future of Rectal Cancer Surgery - A Narrative Review of an International Symposium
}

F. Borja de Lacy, MD. Department of Gastrointestinal Surgery, Institute of Digestive and Metabolic Diseases, Hospital Clinic, Barcelona, Spain

Sami A. Chadi, MD, MSc Division of General Surgery and Surgical Oncology, University Health Network, University of Toronto, Toronto, Ontario, Canada

Mariana Berho MD. Department of Anatomic and Clinical Pathology, Cleveland Clinic, Weston, Florida, USA

Richard J. Heald MD, PhD, FRCS, FACS. The Pelican Cancer Foundation, The Ark, Basingstoke, Hampshire, UK

Jim Khan, MD, MSc, FRCS, Department of Colorectal Surgery, Queen Alexandra Hospital NHS Trust, Portsmouth, UK

Brendan Moran MD, PhD, FRCS. The Pelican Cancer Foundation, The Ark, Basingstoke, Hampshire, UK

Yves Panis, MD, PhD. Department of Colorectal Surgery, Pôle des Maladies de I'Appareil Digestif, Beaujon Hospital, Clichy, France

Rodrigo Perez MD, PhD. Colorectal Department of Gastroenterology, University of Sao Paulo School of Medicine, Sao Paulo, Brazil

Paris Tekkis MD, FRCS. Department of Colorectal Surgery, Croydon University Hospital, Croydon, London, UK

Neil J. Mortensen MD, FRCS. Department of Colorectal Surgery, Churchill Hospital, Oxford University Hospitals, Oxford, UK

Antonio M. Lacy MD, PhD. Department of Gastrointestinal Surgery, Institute of Digestive and Metabolic Diseases, Hospital Clinic, Barcelona, Spain

Steven D. Wexner MD, PhD (Hon), FRCS(Ed), FACS. Digestive Disease Center of the Department of Colorectal Surgery, Cleveland Clinic Florida, Weston, Florida, USA

Manish Chand BSc, MBA, PhD, FRCS. Department of Surgery and Interventional Sciences, The GENIE Centre, University College London UK 


\section{Corresponding author:}

Dr. Manish Chand

Surgery and Interventional Sciences, The GENIE Centre, University College London e-mail: m.chand@ucl.ac.uk

Phone: +44 7956472277 


\begin{abstract}
Surgery remains the mainstay of curative treatment for primary rectal cancer. For mid and low rectal tumors, optimal oncologic surgery requires total mesorectal excision (TME) to ensure the tumor and locoregional lymph nodes are removed. Adequacy of surgery is directly linked to survival outcomes and, in particular, local recurrence. From a technical perspective, the more distal the tumor, the more challenging the surgery and consequently, the risk for oncologically incomplete surgery is higher.

TME can be performed by an open, laparoscopic, robotic or transanal approach. There is a lack of consensus on the 'gold standard' approach with each of these options offering specific advantages. The International Symposium on the Future of Rectal Cancer Surgery was convened to discuss the current challenges and future pathways of the four approaches for TME. This article reviews the findings and discussion from an expert, international panel.
\end{abstract}

\title{
What does this paper add to the existing literature?
}

In the treatment of rectal cancer, an optimal surgical dissection provides improved locoregional control. Which surgical technique is the best option is still under debate. This paper overviews the current state of rectal cancer surgery from an expert point of view, which is always an essential resource for learning. 


\section{INTRODUCTION}

Colorectal cancer is the third most commonly diagnosed malignancy worldwide and the third leading cause of cancer death in the USA. Rectal cancer accounts for approximately one third of all colorectal cancers ${ }^{1}$. Since the early 1990s, total mesorectal excision (TME) has remained the gold standard surgical treatment for rectal cancer following Heald's detailed publication of the technique in $1986^{2}$. In the era of the multidisciplinary approach, the quality of the TME has been shown to be a significant prognostic factor for cancer-specific survival ${ }^{3,4,5}$. The TME technique has evolved from traditional 'open' to minimal access approaches, such as laparoscopic, robotic and more recently, transanal techniques. However, the acceptance of one as better or even equivalent to the others remains a matter of debate. Issues such as case selection, morbidity and recovery, histopathology, oncology, functional outcomes and costs need to be analysed.

On December $9^{\text {th }}$ 2016, The International Symposium on the Future of Rectal Cancer Surgery took place in London. This unique, interactive event was made freely available to viewers around the world through the online educational platform Advances in Surgery (AIS) Channel ${ }^{\circledR}$. A multidisciplinary expert panel of rectal cancer clinicians presented the latest trial data and engaged in discussion for the role of the four different approaches in the treatment of rectal cancer, based on their experience and best evidence. Expert surgical teams performed live simultaneous demonstration of each technique with live commentary from various international experts in attendance. This was also followed by a live macroscopic assessment of the specimens by leading pathologists.

The aim of this article is to provide the reader with an overview of the proceedings of this conference, highlighting the main areas of discussion, debate and controversy in the treatment of patients with rectal cancer.

\section{METHODS}

The coordinating authors (FBDL and SC) invited the panel of experts who had actively participated in the symposium to provide a short summary of each of their presentations. These summaries were merged and edited to account for areas of overlap and redundancy. All authors were subsequently given the opportunity to read 
and edit the merged version of the manuscript to ensure that all expert opinions were appropriately conveyed in the final version.

\section{SYMPOSIUM SUMMARIES}

1. Importance of TME technique: anatomical considerations along the mesorectal plane. RJ Heald

TME was a milestone in the history of cancer surgery as it was the first en-bloc excision for cancer whose boundaries were conceptually and practically founded in embryology. An unexpectedly large improvement in local recurrence rates followed the initial application of the idea of TME to surgical practice - albeit imperfectly implemented in the early stages. This pointed to a fundamental truth, now recognized as relevant to all visceral cancers - that embryologically defined envelopes of tissue with surgical and MRI definable margins and recognizably shiny surfaces, present the surgeon with particular opportunities for cure - exposing the exciting reality that initial cancer spread is often confined to these envelopes following their lymphovascular supply. Despite the importance of a perfect TME being widely understood, mesorectal residues are still the principal source of local recurrence in most countries. Complementary to this "envelope concept" is respect for the surrounding layers and an understanding of their anatomy, not only for more actual "cures" but also for the preservation of the important autonomic functions that we can group together as "Pelvic Happiness" - sexual function, continence, etc. The innermost dissection plane between the covering layer of the gut envelope and the lining layer of the parietes is "holy": the surgeon must constantly follow this innermost plane to achieve an optimal operation. The objective of the newly adopted transanal TME (TaTME) is identical to that for conventional approaches. The first step is to identify the "holy" plane, which is the "innermost dissectable plane". Subsequently, providing traction and counter-traction to identify the white areolar tissue characteristic of an embryological plane allows for the initiation of sharp dissection on the "yellow side of the white" (the innermost mesorectal yellow). When coming from above, identifying this plane is often best achieved around the pedicle above the pelvic brim. Of importance, there are key areas where this dissection must be stressed through various educational aids such as video based education. An example of this is the 
location at which the enveloping fascia can be less clear at the junction with the inferior hypogastric plexus, which has strong implications on erection and ejaculation in males.

All surgeons accept that the deep pelvis, especially in a large male patient, provides the greatest challenge. There are two basic dimensional issues- the difficult areas are far down when approached from above and much closer from below but the angles are also more acute from below. Furthermore it is more difficult to push the tapering mesorectum up from within the encompassing pelvic floor than to draw it gradually out from above whilst opening the planes to be dissected.

The crucial layers low down with potential surgical planes between them are complicated by two septa - Denonvilliers anteriorly, and Waldeyer posteriorly. Denonvilliers' "aponévrose" (as he originally described it in 1835) is a trapezoidal collagenous sheet, tapering medially as it descends, usually adherent to the anterior aspect of the mesorectum and intervening between it and the seminal vesicles 6. Dissection from above is usually in front of Denonvilliers because the plane is easier, and for anterior cancers this must be favoured anyway for a safer margin. If thus incorporated with the specimen, it must then be incised in a $U$ shape to enter the space behind the prostate, carefully preserving the nerves laterally.

Waldeyer is a thickening of the presascral fascia, which has a variable propensity for becoming adherent about half way down the sacrum to the mesorectal fascia, thus potentially dividing the mesorectal/pre-sacral space into two spaces. At this point Waldeyers creates an obstacle for the surgeon dissecting downwards from above and this adherence has been called the Recto-sacral Fascia or Ligament. Faced with this, the abdominal surgeon usually elects to cut through it entering into a space with bare coccyx and the presacral venous plexus. It is important in the transanal approach not to proceed posterior to this layer from below, as it may lead to the lateral compartments with the potential for bleeding, nerve injury, and even dissecting anterior to the prostate, potentially injuring the urethra. As such, it must be emphasized that the "Holy plane" is the innermost dissectable plane that will optimize nerve preservation whilst still delivering perfect TME specimens - all dissected around the "yellow side of the white", i.e. dissection on the mesorectal fat plane leaving behind the white retroperitoneal fascial covering of all the surrounding organs. The 
transanal approach seems to offer a new potential for recognizing and dissecting inside the two layers - Denonvilliers' and Waldeyers' - and thus offering an extra potential for the preservation of autonomic function.

\section{Four approaches for the treatment of rectal cancer: advantages and disadvantages?}

\section{Open TME. B Moran}

The main focus in cancer surgery is to optimize cure and to maximise quantity and quality of life with and minimization of treatment complications. There are particular issues with rectal cancer due to access to the depths of the pelvis and proximity to important surrounding structures such as the genito-urinary systems and the anal sphincters in the restricted space of the bony pelvis. For these reasons there has, and always will be, a conflict between wide excision of the tumor and preservation of tissue to maximise bowel, bladder and sexual function. TME was the real advance in our surgical approach because it balances optimal oncological resection with maximal functional preservation. More recently, optimal imaging by MRI has allowed detailed planning prior to surgery, together with the addition of preoperative radiotherapy in selected cases. The original concept of TME was developed in the era of open surgery and focused on the quality of the specimen assessed by macroscopic appearance (Quirke TME Grading) ${ }^{7}$ and a microscopic assessment of the outermost tumor margin (Quirke Circumferential Resection Margin) ${ }^{8}$, both being prognostic factors for locoregional recurrence ${ }^{3,4}$.

There have been a number of developments in alternative approaches to rectal cancer that capitalize on minimal access techniques and potentially improved visualization of the pelvis. Well-designed prospective comparative studies have failed to consistently demonstrate an advantage to any technique over what remains the standard of care, open surgery ${ }^{9-12}$. Open approaches remain the technique of choice for surgeons who find themselves most effectively able to provide an oncologically sound resection of a rectal cancer, with complete mesorectal excisions, negative circumferential and distal resection margins and a preservation of autonomic nerve function. Additionally, tumors that have a component of adjacent organ, or side-wall, involvement, though nevertheless resectable, are often approached with an open approach given the need for tactile sensation. This allows optimization of intra- 
operative decision making to produce clear margins. In the meantime, technology and concepts have evolved and we are now in the era of laparoscopic, robotic and TaTME. Proponents of robotic and TaTME claim advantages, not only over open surgery, but also compared with laparoscopic surgery. Amongst all of this evolving technology, three statements should be kept in mind: (1) laparoscopic, robotic and TaTME are access techniques and should be classified as "minimal access" in that "minimally invasive" is a misnomer; (2) the focus should be on optimal outcomes which incorporates the appropriate selection of cases, familiarization with technology and surgical expertise with the technique of TME chosen to allow for the goal of optimal specimen quality with clear margins; (3) we should remain cognizant of the fact that the surgeon's technical abilities produce the patient outcomes, not the technique (“Laparoscopes and robots don't operate, surgeons do " ${ }^{13}$ ).

We should embrace new technology, be part of the future, but select the right patient for the right technique and never abandon the gold standard technique of open surgery, nor be afraid to convert a minimal access technique to open if vision, adequate retraction, tactile sensation or other benefits are optimized by an open procedure.

\section{Laparoscopic TME. Y Panis}

Since the early 2000s, laparoscopic resection of colon cancer has been shown to have benefits compared to laparotomy. Long-term oncological safety of colon cancer laparoscopic surgery has also been reported in meta-analyses ${ }^{14}$.

The anatomical restrictions and technical detail of a TME for rectal cancer has meant a longer path to demonstrate the safety of a laparoscopic approach. A laparoscopic approach can provide obvious theoretical advantages with a magnified view allowing a precise dissection in a narrow pelvic space, leading to the possibility of better autonomic nerve sparing strategies. The restrictions of the bony pelvis, the technical expertise necessary to expose the appropriate planes and the associated perioperative, functional and oncologic morbidity of a poor resection have limited the adoption of this technique to subspecialized higher volume surgeons.

In the recent years, the short and long-term results of several randomized clinical trials have been published and demonstrated the safety of a laparoscopic 
approach to TME, with short-term benefits and similar oncological outcomes ${ }^{15,16,17,18}$. However, two recent randomized trials have reported controversial results in this discussion of laparoscopic versus open rectal cancer surgery. These two studies, namely ALaCaRT (Australasian Laparoscopic Cancer of the Rectum) and ACOSOG (American College of Surgeons Oncology Groups) Z6051 were similarly designed noninferiority trials that utilized a new trichotomous composite outcome based on the completeness of the mesorectal excision as well as the negativity of the circumferential and distal resection margins ${ }^{19,20}$. The lack of validation, and therefore the unestablished clinical and oncologic significance of this primary outcome, has introduced some hesitance in the oncology community in accepting these trials results, given that both trials failed to demonstrate a non-inferiority of the laparoscopic approach relative to the traditional open approach. The implications that these pathologic outcomes will have on survival data are expected to be reported in future publications from these groups. It can be seen that meta-analyzing these trials would be difficult to interpret given the different designs as well as the differing primary outcomes and therefore ways in which trials were powered. In table 1 , the most important outcomes of the above mentioned randomized clinical trials are reported. Recently, a meta-analysis of 27 studies and 10861 patients found no difference in terms of rate of complete resection, mesorectal excision quality and local recurrence between laparoscopic and open TME ${ }^{21}$.

Laparoscopic TME results in enhanced recovery compared with open TME ${ }^{15}$. Nevertheless, in the COLOR II trial neither the health-related quality of life nor the genitourinary dysfunction were improved by the laparoscopic approach ${ }^{22}$, suggesting that the clinical benefits of laparoscopy persist only in the short postoperative period.

\section{Robotic TME. J Khan}

Despite the advantages of laparoscopic surgery, the technique has its own limitations with a two-dimensional view, limitations in range of movements, fulcrum effects of various bony aspects of the pelvis resulting in problems in access and exposure, especially in the pelvic depths of patients with large tumors and obese body habitus. All of this leads to an extended learning curve for the surgeon. 
The robotic approach seems to be a valid alternative to combat some of the issues encountered with laparoscopy. Three-dimensional views, endowristed instrumentation and a stable camera platform make it a very attractive option for the surgeon. This may translate into improved tissue dissection, accurate cancer clearance, precision surgery with minimal collateral damage and excellent functional outcomes for patients. For an experienced laparoscopic colorectal surgeon the learning curve for robotics is minimal, and can be as little as $10-15$ cases $^{23}$. Operative costs and reports of longer operative times have been the two main deterrents for the uptake of this technology amongst many colorectal surgeons.

A robotic approach to the pelvis for rectal cancer is feasible and safe, and the initial results are very encouraging ${ }^{24}$. Large series from high volume centres might provide the evidence for its efficacy and cost effectiveness. The preservation of sexual, urological and bowel function, and decreased fatigue and stress for the surgeon, are going to be major stand out points encouraging surgeons to embrace this technology for the management of rectal cancer.

Provisional results from the multi-national ROLARR trial found no difference in pathological circumferential resection margin (pCRM) positive rate $(5.1 \%$ in the robotic group vs. $6.3 \%$ in the laparoscopic group (OR $0.79,95 \% \mathrm{Cl} 0.35-1.76)$ ) and postoperative complications (33.1\% vs. $31.7 \%)^{25}$. Anastomotic complications occurred in $2.6 \%$ and $3.0 \%$ of laparoscopic and robotic cases respectively. Thirty-day mortality was less than $1 \%$ in both groups. Conversion to open, which was the primary outcome for this study, was necessary in $8.1 \%$ for robotic and $12.3 \%$ for laparoscopic TMEs ( $p=$ 0.16). A priori subgroup analysis showed fewer rate of conversion in men and obese patients undergoing robotic resection, suggesting a benefit for the robotic TME in these cohorts of patients.

\section{Transanal TME. AM Lacy}

Performance of an optimal TME mainly depends on the anatomy of the patient, tumor characteristics, response to neoadjuvant therapy and a composite of surgical technique and surgical team skills. Working in the low pelvis, especially in male and obese patients, increases the difficulty of the procedure and threatens the fulfilment of a complete mesorectal excision with negative margins. This fact might restrict the 
quality of the TME when it is approached transabdominally, but could be less challenging when approached transanally. Potentially, TaTME allows for an increased quality of TME in mid and low rectal tumors, together with a lower rate of abdominal conversion and permanent stoma. Whiteford et al were the first to performing a natural-orifice transanal endoscopic rectosigmoid resection in a human cadaver ${ }^{26}$, while Atallah and colleagues established the feasibility and safety of transanal minimally invasive surgery (TAMIS) ${ }^{27}$. This set the stage for a cadaveric series that was the premise for the performance of the first TaTME in Barcelona ${ }^{28}$.

TaTME can be performed either by one-team or a two-team approach, the latter being called the "Cecil approach" ${ }^{29}$. Where TaTME is to be performed by a oneteam approach starting with the abdominal phase is often recommended in order to avoid retropneumoperitoneum that might make the laparoscopic resection harder. The two-team approach is strongly advocated by the TaTME community. It allows for shorter operative times, improved visualization, better traction and counter-traction while facilitating the ultimate goal of this approach in optimizing the oncologic outcomes and functional preservation in rectal cancer surgery ${ }^{29,30}$. Three-dimensional cameras and insufflators with continuous flow and smoke evacuation have further optimized the safety and quality of these resections. However, potentially the most important step of the transanal approach is a correct closure of the bowel lumen so as to follow an adequate plane of mesorectal dissection. This technique is a high-stakes procedure with multiple potential pitfalls and complications, both functionally and oncologically. As such, it is strongly recommended that surgeons ensure they have an adequate expertise in laparoscopic and transanal surgery, following an established training process ${ }^{31}$ and ensure that early experiences are proctored for optimal collegial support.

Data from the international TaTME registry showed pCRM involvement in $2.4 \%$ of cases. Composite poor pathological outcome was seen in $7.4 \%$. Early anastomotic leaks occurred in $6.7 \%$. Post operative morbidity was $32.6 \%$ and the mortality rate was 2.6\%. Conversion was necessary in $6.3 \%$ during the abdominal part of the procedure and $2.8 \%$ for the perineal approach ${ }^{32}$. The data suggests that TaTME is feasible, safe, and could result in improved oncological outcomes compared to conventional approaches to TME. 


\section{Do the results of TME vary by technique? M Berho}

One of the most important functions of the pathologist at the time of examining rectal cancer specimens is that of an auditor. It has been shown that certain macroscopic features actually reflect the quality of the surgery performed. These features can be easily recognized by most pathologists at the time of gross examination and include (1) the integrity of the mesorectum, (2) the status of the resected margins and (3) the number of dissected lymph nodes.

Integrity of the mesorectum: the integrity of the mesorectum is directly related to the plane at which the surgeon performs dissection of the rectum and the perirectal soft tissue from the pelvis, thus the plane of surgery can be mesorectal, intramesorectal, or muscularis propria as delineated by Quirke and colleagues ${ }^{7}$ (Figure 1 ).

Resection margins: the concept of distal tumor spread is presence of tumor cells within the mesorectum or rectum, distal to the level of the tumor. Several studies demonstrated that the presence of tumor distal spread beyond $2 \mathrm{~cm}$ is exceedingly rare ${ }^{33,34}$. As a result of this discovery, distal margins of $2 \mathrm{~cm}$ became generally accepted. More recently, with the introduction of surgical techniques such as double stapling as well as neoadjuvant chemoradiation (nCRT) and the widespread use of TME, $1 \mathrm{~cm}$ or sub-centimetre distal margins have gained greater approval. The importance of the CRM has been demonstrated by Quirke in a landmark study published in 1986, where the high incidence of local recurrence in patients with rectal cancer was directly related to the involvement of the circumferential (radial) margin of resection (CRM) rather than the distal margin ${ }^{4}$. Numerous subsequent studies have confirmed that the presence of tumor $\leq 1 \mathrm{~mm}$ from the CRM adversely affects outcomes both at the local and systemic levels. There are three basic mechanisms of CRM involvement: 1) direct tumor extension; 2) foci of vascular/perineural invasion or tumor deposit; and 3) positive lymph node. Although there are currently no published series addressing the prognostic significance of each of these events, it may appear that direct extension of the tumor into the CRM would carry a more ominous prognosis. Several factors have been associated with a positive CRM including large 
and deep tumors, vascular and perineural invasion, poor tumor differentiation, advanced age, and mesorectal quality based on the quality of the surgical resection. The latter of these variables has a significant influence in the status of the CRM, logically; those specimens with incomplete peritumoral mesorectums have a higher risk of a positive CRM.

Lymph node evaluation: although the adverse impact of lymph node metastasis is well known, the total number of dissected lymph nodes, regardless of the status (positive or negative), also influences the outcome of patients with colorectal cancer; the reasons for this remains unclear. It is important to point out that the rectum inherently contains fewer and smaller lymph nodes compared to other segments of the intestinal tract. In addition, factors including obesity, male gender, advanced age and neoadjuvant radiation have all been associated with a decreased number of lymph nodes ${ }^{35}$. Conversely, tumor features such as depth of invasion into the rectal wall and poor differentiation have been correlated with higher node yield. Certainly, the number of lymph nodes evaluated also reflects the quality of the surgery performed by the surgeon as well as the diligence and effort of the dissecting pathologist. In cases of an optimal TME, the lymph node harvest depends entirely on meticulous work by the pathologist. Although lymph node dissection from rectal cancer specimens is traditionally carried out by a combination of palpation and visualization of the mesorectal tissue, several auxiliary techniques have been developed to further increase nodal yields.

\section{Choosing the optimal technique to optimize patient outcomes. P Tekkis}

Over time, PCRM involvement and conversion rates in laparoscopic surgery appear to have decreased. These have become the new benchmark in rectal cancer surgery. The improvements are related to advances in neoadjuvant treatment as well as surgical techniques. Likewise, reduction in clinical anastomotic leak rates may be the result of better patient selection for defunctioning stoma in addition to improved stapling devices and assessments of anastomotic perfusion. Short-term morbidity and mortality rates have remained the same, regardless of surgical approach. This may reflect the inherent challenges posed by patient and cancer characteristics that could not be 
mitigated by evolving of surgical techniques.

Existing level-1 evidence has suggested that laparoscopic, robotic and transanal approaches to TME are safe alternatives to open surgery. This evidence does not help the surgeon decide on the most appropriate approach to ensure best clinical outcomes for an individual patient. It is important to note that other factors affect clinical outcomes in rectal cancer. The Mercury II study showed that tumor characteristics greatly influence the risk of $\mathrm{pCRM}$ involvement ${ }^{36}$. In a cohort of patients predominantly undergoing open TME, pCRM was positive in $9.0 \%$ of cases. This varied significantly based on tumor features on MRI: extra mural vascular invasion, anterior tumors, low tumors, and tumors involving "at risk" planes. The risk of pCRM involvement ranged from $2.27 \%$ in tumors without risk factors to $53 \%$ when all four risk factors were present.

Evidence is needed to help the surgeon select the best surgical technique for each case based on patient and tumor characteristics. For example, does the robotic approach offer most benefit for upper rectal cancer in obese male patients? Or is TaTME the best approach to low rectal cancer in obese individuals? Novel approaches to future surgical study designs are required to answer these questions.

Surgeons will need to use their best judgment in choosing the correct technique based on their technical skill set, their knowledge of their own audited results and the patient/tumor specific factors. Despite the holy grail of randomized trials, not all patients or tumors are equivalent, thereby necessitating a tailored approach that suits the needs of each specific patient presentation. As can be seen from above, there is a paucity of data that allows for such conclusions to be made. Despite this fact, we must continue to observe our own outcomes to guide the best oncologic outcome of our selected technique.

\section{Non-operative approaches. "Watch and Wait" - The Outsider. R Perez}

Neoadjuvant chemoradiation has been shown to result in complete eradication of rectal cancer in the resected specimen in selected patients, known as complete pathological response ( $P C R$ ). In this context patients with a $\mathrm{pCR}$ have had excisional surgery and would have undergone considerable morbidity, functional consequences and frequent need for temporary or permanent stomas even though the specimen 
obtained after resection had no residual cancer ${ }^{37}$. In order to avoid the potentially unnecessary consequences of radical surgery, attempts were made to identify patients with a complete clinical response (cCR) prior to radical surgery, and offer them an organ-preserving strategy ${ }^{38}$. After identification of the clinical features of $\mathrm{CCR}$ (whitening of the mucosa, teleangiectasia and the absence of residual ulceration, mass or stenosis) patients can be enrolled in a strict follow-up program also known as the "Watch and Wait" strategy ${ }^{39}$. Initial clinical assessment of response is made at least eight weeks after finishing nCRT, allowing for enough time for tumor regression. At MRI, a low-signal intensity of the area harbouring the initial cancer (used as an estimate to tumor regression grade - mrTRG1) would be consistent with $\mathrm{CCR}^{40,41}$.

The oncological outcomes of the "Watch and Wait" approach in selected patients have been shown to be at least similar to radical surgery, but with improved functional outcomes, lesser risk for a stoma and perhaps even improved overall survival 38, 42, 43. However, clinical and radiological identification of such patients remains challenging with frequent early local recurrences (or tumor regrowths) underscoring the importance of close surveillance ${ }^{43,44}$. Even so, recurrences have been reported to be mostly within the rectal wall (and rarely exclusively mesorectal), frequently amenable to salvage resection and general considered without oncological compromise compared with initial surgery ${ }^{44}$.

Considering that baseline features may ultimately influence the chances of developing a cCR, restricting the use of $\mathrm{nCRT}$ to advanced disease ( $\mathrm{mrCRM}+, \mathrm{mrEMVI+}$ or mrN2) will also restrict the number of patients that could potentially benefit from avoiding radical surgery, particularly in the setting of abdominoperineal excision (APE) resections ${ }^{45}$. Therefore, considering the opportunity for organ-preservation after a cCR, nCRT may be an attractive alternative for less advanced cancers such as mrT2NOin the most distal part of the rectum, where an APE is deemed necessary for "lowness" of the tumor. However, considering the current inability to predict response based on pre-treatment biopsies, partly due to significant intratumoral heterogeneity, the potential benefits of "Watch and Wait" need to be weighted against the potential disadvantages of TME after nCRT in the setting of incomplete response in a patient with early baseline staged tumor which might have been amenable to curative surgical excision ${ }^{46}$. 


\section{Attempts to reduce anastomotic leaks. SD Wexner}

Despite many improvements in the quality of rectal cancer surgery, anastomotic leak rates remain between 10 and $15 \% 47,48$. The adverse sequela of such leaks is formidable and costly. In the immediate post-operative period, additional medical and surgical procedures prolong hospitalization and increase expense. One recent study revealed a six-fold increase in 30-day mortality following anastomotic leak ${ }^{49}$. Longterm morbidity includes impaired function, an increased incidence of permanent stoma, increased rates of local recurrence and decreased long-term survival.

While faecal diversion has been shown to decrease the severity of leaks and possibly even decrease the incidence of leaks, stomas are associated with their own attendant morbidity including dehydration and complications following stoma reversal. Recent attempts to decrease the incidence of anastomotic leaks have included alternate types of anastomosis such as colonic J pouch and side-to-side (Baker) anastomosis as well as improvements in anastomotic technique with compression anastomosis. Several publications have clearly demonstrated a very low incidence of leak following compression anastomosis ${ }^{50,51}$. Other novel approaches have been internal bypass and anastomotic sealants 52, 53. Two large multicentre randomized controlled trials failed to show any benefit to anastomotic buttressing ${ }^{54}$, 55. The most promising currently available option is Indocyanine Green (ICG) fluorescence angiography ${ }^{56}$. Multiple studies have noted that this inexpensive, safe, and reliable technique has changed the decision on the proximal resection margin in $5 \%-8 \%$ of patients and such a strategy been followed by leak rates of $1.4 \%-4 \%{ }^{57,58}$. Because of these impressive results, many surgeons routinely use ICG imaging for all distal anastomoses.

\section{The future of surgical training. SA Chadi}

As has been highlighted, rectal cancer surgery represents a high stakes procedure with perioperative, functional and oncologic implications to inappropriate technique. Many learning curve analyses have suggested that it can take well over 50 procedures to reach a safe level of competence, even in the most expert of hands. Various society endorsed platforms exist to promote new surgical technique such as the Fundamentals 
of Laparoscopic Sugery ${ }^{\circledR}$ and the LAPCO curriculum. The former has now become a requirement for graduation from North American surgical residency programs. The LAPCO curriculum is a structured curriculum that allowed for the safe introduction of laparoscopic colorectal surgery in the practices of established consultant surgeons.

These prior experiences with new surgical techniques have prompted the surgical community to advocate for the safe introduction of complex procedures such as robotic surgery and more recently, TaTME. Multiple international courses exist for this technique, with some run by surgical societies. These courses, as well as some of the world's highest volume TaTME surgeons, have advocated for minimal annual rectal cancer volumes as well as a demonstrated expertise in laparoscopic and transanal surgery prior to embarking on this new technique. Additionally, a number of interactive educational platforms such as the AIS Channel ${ }^{\circledR}$ and the iLappSurgery Foundation ${ }^{\circledR}$ have provided additional sources of educational resources through the availability of live and previously recorded procedures as well as the availability of didactic resources, in mobile formats.

These tenants of surgical education and implementation of new techniques have also led to the establishment of proctorship programs to encourage newly adopting surgeons to seek the assistance of experienced colleagues so as not to compromise the oncologic outcomes of patients. Through ethical implementation methods, these new techniques can be safely introduced into the practice of subspecialty surgeons to further enhance the functional and oncologic outcomes of patients.

\section{CONCLUSION}

The International Symposium on the Future of Rectal Cancer Surgery provided a singularly unique educational experience for a live global audience of more than 25.000 viewers from over 100 countries. Combining expert presentations and evidence from all members of the multidisciplinary team including the latest and most controversial data with simultaneous live demonstration of the four approaches allowed 'virtual' attendees to directly engage in an evolving educational event. By using the AIS Channel ${ }^{\circledR}$ platform, viewers were encouraged to challenge the 'experts' 
and participate in a live global debate. This inclusive approach meant that huge areas of rectal cancer surgery were discussed, thus enriching the content.

The data presented, complemented by the live surgery, eloquently demonstrated the principles of rectal cancer surgery and how regardless of the approach, one must strive for oncological success whilst respecting the functional anatomical consequences of pelvic surgery. Each approach, in appropriately experienced hands, can lay claim to this but the over-riding message conveyed by all participants was that it is unlikely that there is one approach that is ideal for all situations. The primary outcome measures used to explain the benefits of surgery in the majority of the presentations included some description of the quality of the surgical specimen, complication rates and survival data e.g. overall survival and disease-free survival. The quality of excision is a near-immediate quality assurance measure and a validated outcome measure for survival outcomes ${ }^{5}$. Given this background, there are specific conditions (patient or tumor-related) that may give one approach advantage over another e.g. tumor size, distance from the anal verge, patient body mass index. Furthermore, availability of resources may be a further consideration where the cost of the procedure may preclude certain approaches and direct surgeons towards others.

The original principles of TME were historically described in the context of open surgery and are still considered by most surgeons as the gold standard by which all innovative techniques are measured. However, technology has allowed improved vision and dexterity beyond the limitations of the human eye and hand, respectively. It is not unreasonable to try and develop technology to improve the status quo, but this must be done in a safe and controlled manner through peer-review, safety and quality assurance. Many of the criticisms directed at robotics and TaTME were similarly directed at laparoscopy when first introduced, but it is important to appreciate that modern-day surgery has never been under such scrutiny, and the governance surrounding new techniques is far more rigid than at the time of Heald's original description of TME.

Technology will continue to influence the future of surgery but it is unlikely that surgeons will be equally adept at all techniques and approaches. This may mean that these four techniques become centralised with patients being directed to a surgeon 
proficient in one technique rather than another. However, whichever technique is employed the underlying principles of cancer surgery must not be lost and this must be the gold standard rather than fanaticism for the actual approach. 


\section{AUTHOR CONTRIBUTIONS}

Study conception and design: de Lacy, Chadi, Chand

Acquisition of data: de Lacy, Chadi

Drafting of manuscript: de Lacy, Chadi, Berho, Heald, Khan, Moran, Panis, Perez, Tekkis, Lacy, Wexner, Chand

Critical revision: de Lacy, Chadi, Berho, Heald, Khan, Moran, Panis, Perez, Tekkis, Mortensen, Lacy, Wexner, Chand 


\section{REFERENCES}

1. Cancer facts and figures 2014. Atlanta: American Cancer Society. http://www.cancer.org/acs/groups/content/@research/documents/webcontent/acsp c-042151.pdf. Published 2014. Accessed January 20, 2017

2. Heald RJ, Ryall RD. Recurrence and survival after total mesorectal excision for rectal cancer. Lancet. 1986; 1(8496):1479-82

3. Kapiteijn E, Marijnen CA, Colenbrander AC, et al. Local recurrence in patients with rectal cancer diagnosed between 1988 and 1992: A population-based study in the west Netherlands. Eur J Surg Oncol. 1998; 24: 528,1998-535

4. Quirke P, Durdey P, Dixon MF, Williams NS. Local recurrence of rectal adenocarcinoma due to inadequate surgical resection: histopathological study of lateral tumor spread and surgical excision. Lancet. 1986;2:996-9

5. Quirke P, Steele R, Monson J, et al. Effect of the plane of surgery achieved on local recurrence in patients with operable rectal cancer: a prospective study using data from the MRC CR07 and NCICCTG CO16 randomised clinical trial. Lancet. 2009; 373(9666):821-8

6. Lindsey I, Warren BF, Mortensen NJ. Denonvilliers' fascia lies anterior to the fascia propria and rectal dissection plane in total mesorectal excision. Dis Colon Rectum. $2005 ; 48(1): 37-42$

7. Nagtegaal ID, van de Velde CJ, van der Worp E, Kapiteijn E, Quirke P, van Krieken JH. Macroscopic evaluation of rectal cancer resection specimen: clinical significance of the pathologist in quality control. J Clin Oncol. 2002; 20:1729-34 
8. Nagtegaal ID, Quirke P. What is the role for the circumferential margin in the modern treatment of rectal cancer? J Clin Oncol. 2008; 26(2):303-12. doi: $10.1200 / J C O .2007 .12 .7027$

9. Kuhry E, Schwenk WF, Gaupset R, et al. Long-term results of laparoscopic colorectal cancer resection. Cochrane Database Syst Rev. 2008; CD003432

10. Row D, Weiser MR. An update on laparoscopic resection for rectal cancer. Cancer Control. 2010; 17: 16-24

11. Jayne DG, Thorpe HC, Copeland J, et al. Five-year follow-up of the Medical Research Council CLASICC trial of laparoscopically assisted versus open surgery for colorectal cancer. Br J Surg. 2010; 97: 1638-45

12. Huang MJ, Liang JL, Wang $\mathrm{H}$, et al. Laparoscopic-assisted versus open surgery for rectal cancer: a meta-analysis of randomized controlled trials on oncologic adequacy of resection and long-term oncologic outcomes. Int J Colorectal Dis. 2011; 26: 415-21

13. Moran B. Primacy of surgery in cancer care: a global situation. Lancet Oncol. 2015; 16(11):1193-224

14. Athanasiou CD, Robinson J, Yiasemidou M, et al. Laparoscopic vs open approach for transverse colon cancer. A systematic review and meta-analysis of short and long term outcomes. Int J Surg. 2017; 41:78-85. doi: 10.1016/j.ijsu.2017.03.050

15. van der Pas $M H$, Haglind E, Cuesta MA, et al. Laparoscopic versus open surgery for rectal cancer (COLOR II): short-term outcomes of a randomised, phase 3 trial. Lancet Oncol. 2013; 14:210-218

16. Kang SB, Park JW, Jeong SY, et al. Open versus laparoscopic surgery for mid or low rectal cancer after neoadjuvant chemoradiotherapy (COREAN trial): short-term 
outcomes of an open-label randomised controlled trial. Lancet Oncol. 2010; 11:637645

17. Bonjer HJ, Deijen $\mathrm{CL}$, Abis $\mathrm{GA}$, et al. A randomized trial of laparoscopic versus open surgery for rectal cancer. N Engl J Med. 2015; 2;372(14):1324-32

18. Jeong SY, Park JW, Nam BH, et al. Open versus laparoscopic surgery for mid-rectal or low-rectal cancer after neoadjuvant chemoradiotherapy (COREAN trial): survival outcomes of an open-label, non-inferiority, randomised controlled trial. Lancet Oncol. 2014; 15(7):767-74

19. Fleshman J, Branda M, Sargent DJ, et al. Effect of Laparoscopic-Assisted Resection vs Open Resection of Stage II or III Rectal Cancer on Pathologic Outcomes. The ACOSOG Z6051 Randomized Clinical Trial. JAMA. 2015; 314(13):1346-1355

20. Stevenson ARL, Solomon MJ, Lumley JW, et al. Effect of Laparoscopic-Assisted Resection vs Open Resection on Pathological Outcomes in Rectal Cancer. The ALaCaRT Randomized Clinical Trial. JAMA. 2015; 314(13):1356-1363

21. Arezzo A, Passera R, Salvai A, et al. Laparoscopy for rectal cancer is oncologically adequate: a systematic review and meta-analysis of the literature. Surg Endosc. 2015; 29:334-348

22. Andersson J, Abis G, Gellerstedt $M$, et al. Patient-reported genitourinary dysfunction after laparoscopic and open rectal cancer surgery in a randomized trial (COLOR II). Br J Surg. 2014; 101:1272-1279

23. Odermatt $M$, Ahmed J, Panteleimonitis $S$, Khan J, Parvaiz A. Prior experience in laparoscopic rectal surgery can minimise the learning curve for robotic rectal resections: A cumulative sum analysis. Surg Endosc. 2017; 31(10):4067-4076. doi: $10.1007 / \mathrm{s} 00464-017-5453-9$ 
24. Ahmed J, Nasir M, Flashman K, Khan J, Parvaiz A. Totally robotic rectal resection: an experience of the first 100 consecutive cases. Int J Colorectal Dis. 2016; 31(4): 869-76. doi: $10.1007 / \mathrm{s} 00384-016-2503-z$

25. Collinson FJ, Jayne DG, Pigazzi A, et al. An international, multicentre, prospective, randomised, controlled, unblinded, parallel-group trial of robotic-assisted versus standard laparoscopic surgery for the curative treatment of rectal cancer. Int $J$ Colorectal Dis. 2012; 27(2):233-241

26. Whiteford MH, Denk PM, Swanstrom LL. Feasibility of radical sigmoid colectomy performed as natural orifice translumenal endoscopic surgery (NOTES) using transanal endoscopic microsurgery. Surg Endosc. 2007; 21:1870-1874

27. Atallah S, Larach S, Albert M. Transanal minimally invasive surgery: a giant leap forward. Surg Endosc. 2010; 24:2200-2205

28. Sylla P, Rattner DW, Delgado S, Lacy AM. NOTES transanal rectal cancer resection using transanal endoscopic microsurgery and laparoscopic assistance. Surg Endosc. 2010; 24:1205-1210.

29. Arroyave MC, DeLacy FB, Lacy AM. Transanal Total Mesorectal Excision (TaTME) for Rectal Cancer: Step by step description of the surgical technique for a two-teams approach. EJSO. 2016; pii: S0748-7983(16)30964-7. doi: 10.1016/j.ejso.2016.10.024

30. Koedman TW, van Ramshorst GH, Deijen CL, et al. Transanal total mesorectal excision (TaTME) for rectal cancer: effects on patient-reported quality of life and functional outcome. Tech Coloproctol 2017; 21: 25-33

31. Francis N, Penna M, Mackenzie H, Carter F, Hompes R. Consensus on structured training curriculum for transanal total mesorectal excision (TaTME). Surg Endosc. 2017; 31(7):2711-2719. doi: 10.1007/s00464-017-5562-5 
32. Penna M, Hompes R, Arnold S, et al. Transanal Total Mesorectal Excision: International Registry Results of the First 720 Cases. Ann Surg. 2016; 266(1):111-117. doi: 10.1097/SLA.0000000000001948

33. Grinell RS. Distal intramural spread of carcinoma of the rectum and rectosigmoid. Surg Gynecol Obstet. 1954; 99(4):421-430

34. Andreola S, Leo E, Belli F, et al. Distal Intramural Spread in Adenocarcinoma of the Lower Third of the Rectum Treated with Total Rectal Resection and Coloanal Anastomosis. Dis Colon Rectum. 1997; 40(1):25-29

35. Amajoyi R, Lee $\mathrm{Y}$, Recio PJ, Kondylis PD. Neoadjuvant therapy for rectal cancer decreases the number of lymph nodes harvested in operative specimens. Am J Surg. 2013; 205(3):289-92; discussion 292. doi: 10.1016/j.amjsurg.2012.10.020

36. Battersby NJ, How P, Moran B, et al. Prospective Validation of a Low Rectal Cancer Magnetic Resonance Imaging Staging System and Development of a Local Recurrence Risk Stratification Model: The MERCURY II Study. Ann Surg. 2016; 263: 751-760

37. Smith FM, Rao C, Oliva Perez R, et al. Avoiding radical surgery improves early survival in elderly patients with rectal cancer, demonstrating complete clinical response after neoadjuvant therapy: results of a decision-analytic model. Dis Colon Rectum. 2015; 58(2):159-71

38. Habr-Gama A, Perez RO, Nadalin W, et al. Operative Versus Nonoperative Treatment for Stage 0 Distal Rectal Cancer Following Chemoradiation Therapy. Ann Surg. 2004; 240(4):711-7

39. Habr-Gama A, Perez RO, Wynn G, Marks J, Kessler H, Gama-Rodrigues J. Complete Clinical Response After Neoadjuvant Chemoradiation Therapy for Distal Rectal Cancer: Characterization of Clinical and Endoscopic Findings for Standardization. Dis Colon Rectum. 2010; 53(12):1692-8 
40. Patel UB, Brown G, Rutten $\mathrm{H}$, et al. Comparison of magnetic resonance imaging and histopathological response to chemoradiotherapy in locally advanced rectal cancer. Ann Surg Oncol. 2012; 19(9):2842-52

41. Bhoday J, Smith F, Siddiqui MR, et al. Magnetic Resonance Tumor Regression Grade and Residual Mucosal Abnormality as Predictors for Pathological Complete Response in Rectal Cancer Postneoadjuvant Chemoradiotherapy. Dis Colon Rectum. 2016; 59(10):925-33

42. Maas M, Beets-Tan RGH, Lambregts DM, et al. Wait-and-See Policy for Clinical Complete Responders After Chemoradiation for Rectal Cancer. J Clin Oncol. 2011; 29(35):4633-40

43. Renehan AG, Malcomson L, Emsley R, et al. Watch-and-wait approach versus surgical resection after chemoradiotherapy for patients with rectal cancer (the OnCoRe project): a propensity-score matched cohort analysis. Lancet Oncol. 2016; 17(2):17483

44. Habr-Gama A, Gama-Rodrigues J, São Julião GP, et al. Local recurrence after complete clinical response and watch and wait in rectal cancer after neoadjuvant chemoradiation: impact of salvage therapy on local disease control. Int J Radiat Oncol Biol Phys. 2014; 88(4):822-8

45. Habr-Gama A, Sabbaga J, Gama-Rodrigues J, et al. Watch and Wait Approach Following Extended Neoadjuvant Chemoradiation for Distal Rectal Cancer. Dis Colon Rectum. 2013; 56(10):1109-17

46. Bettoni F, Masotti C, Habr-Gama A, et al. Intratumoral Genetic Heterogeneity in Rectal Cancer. Ann Surg. 2017; 265(1):e4-e6 
47. Chadi SA, Fingerhut A, Berho M, et al. Emerging Trends in the Etiology, Prevention, and Treatment of Gastrointestinal Anastomotic Leakage. J Gastrointest Surg. 2016; 20(12):2035-2051

48. Vallance $A$, Wexner $S$, Berho $M$, et al. A collaborative review of the current concepts and challenges of anastomotic leaks in colorectal surgery. Colorectal Dis. 2017; 19(1):01-012. doi: 10.1111/codi.13534

49. Tevis SE, Carchman, EH, Foley EF, Heise CP, Harms BA, Kennedy GD. Does anastomotic leak contribute to high failure-to-rescue rates? Ann Surg. 2016; 263:11481151

50. Masoomi H, Luo R, Mills S, Carmichael JC, Senagore AJ, Stamos MJ. Compression anastomosis ring device in colorectal anastomosis: a review of 1,180 patients. Am J Surg. 2014; 205(4):447-51. doi: 10.1016/j.amjsurg.2012.03.013

51. D'Hoore A, Albert MR, Cohen SM, et al. COMPRES: a prospective postmarketing evaluation of the compression anastomosis ring CAR $27\left(^{\mathrm{TM}}\right) /$ ColonRing $\left({ }^{\mathrm{TM}}\right)$. Colorectal Dis. 2015; 17(6):522-9. doi: 10.1111/codi.12884

52. Ye F, Wang D, Xu X, Liu F, Lin J. Use of Intracolonic Bypass Secured by a Biodegradable Anastomotic Ring to Protect the Low Rectal Anastomosis. Dis Colon Rectum. 2008; 51(1):109-115

53. Huh JW, Kim HR, Kim YJ. Anastomotic leakage after laparoscopic resection of rectal cáncer: the impact of fibrin glue. Am J Surg. 2010; 199(4):435-441

54. Senagore A, Lane FR, Lee E, et al. Bioabsorbable staple line reinforcement in restorative proctectomy and anterior resection: a randomized study. Dis Colon Rectum. 2014; 57(3):324-30. doi: 10.1097/DCR.0000000000000065 
55. Placer C, Enríquez-Navascués JM, Elorza G, et al. Preventing complications in colorectal anastomosis: results of a randomized controlled trial using bioabsorbable staple line reinforcement for circular stapler. Dis Colon Rectum. 2014; 57(10):1195201. doi: 10.1097/DCR.0000000000000207

56. Keller D, Ishizawa T, Cohen R, Chand M. Indocyanine green fluorescence imaging in colorectal surgery: overview, applications, and future directions. Lancet Gastroenterol Hepatol. 2017; 2(10):757-766. doi: 10.1016/S2468-1253(17)30216-9

57. James DR, Ris F, Yeung TM, et al. Fluorescence angiography in laparoscopic low rectal and anorectal anastomoses with pinpoint perfusion imaging--a critical appraisal with specific focus on leak risk reduction. Colorectal Dis. 2015; Oct;17 Suppl 3:16-21. doi: 10.1111/codi.13033

58. Jafari MD, Wexner SD, Martz JE, et al. Perfusion assessment in laparoscopic leftsided/anterior resection (PILLAR II): a multi-institutional study. J Am Coll Surg. 2015; 220(1):82-92.e1. doi: 10.1016/j.jamcollsurg.2014.09.015 


\begin{tabular}{|c|c|c|c|c|c|}
\hline \multirow{2}{*}{\multicolumn{2}{|c|}{ Year }} & COREAN & COLOR II & $\begin{array}{c}\text { ACOSOG } \\
\text { Z6051 }\end{array}$ & ALaCaRT \\
\hline & & 2006-2009 & $2003-2010$ & $2008-2013$ & $2010-2014$ \\
\hline \multirow{3}{*}{ Positive pCRM* } & Open & $4.1 \%$ & $10.0 \%$ & $12.1 \%$ & $3.0 \%$ \\
\hline & Laparoscopic & $2.9 \%$ & $9.5 \%$ & $7.7 \%$ & $6.8 \%$ \\
\hline & $p$ & n.s. & n.s. & n.s & n.s. \\
\hline \multirow{3}{*}{ Complete TME** } & Open & $74.7 \%$ & $91.5 \%$ & $95.1 \%$ & $91.9 \%$ \\
\hline & Laparoscopic & $72.4 \%$ & $88.4 \%$ & $92.1 \%$ & $86.5 \%$ \\
\hline & $\mathrm{p}$ & n.s. & n.s. & n.s. & \\
\hline \multirow{3}{*}{ 30-day morbidity } & Open & $23.5 \%$ & $37.1 \%$ & $58.1 \%$ & - \\
\hline & Laparoscopic & $21.2 \%$ & $39.8 \%$ & $57.1 \%$ & - \\
\hline & $p$ & n.s. & n.s. & n.s. & n.s \\
\hline \multirow{3}{*}{ Anastomotic Leak } & Open & $0.0 \%$ & $10.4 \%$ & $2.1 \%$ & $3.4 \%$ \\
\hline & Laparoscopic & $1.2 \%$ & $12.5 \%$ & $2.3 \%$ & $2.9 \%$ \\
\hline & $\mathrm{p}$ & n.s & n.s. & n.s. & n.s. \\
\hline \multirow{3}{*}{ 30-day mortality } & Open & $0.0 \%$ & $1.7 \%$ & $0.9 \%$ & $0.8 \%$ \\
\hline & Laparoscopic & $0.0 \%$ & $1.1 \%$ & $0.8 \%$ & $0.4 \%$ \\
\hline & $\mathrm{p}$ & n.s. & n.s. & n.s. & n.s. \\
\hline Conversion & & $1.2 \%$ & $17.4 \%$ & $11 \%$ & $9 \%$ \\
\hline 3-year locoregional & Open & $4.9 \%$ & $5.0 \%$ & - & - \\
\hline
\end{tabular}




\begin{tabular}{|c|c|c|c|c|c|}
\hline \multirow[t]{2}{*}{ recurrence } & Laparoscopic & $2.6 \%$ & $5.0 \%$ & - & - \\
\hline & $\mathrm{p}$ & n.s. & n.s. & - & - \\
\hline \multirow{3}{*}{$\begin{array}{l}\text { 3-year disease-free } \\
\text { survival }\end{array}$} & Open & $72.5 \%$ & $70.8 \%$ & - & - \\
\hline & Laparoscopic & $79.2 \%$ & $74.8 \%$ & - & - \\
\hline & $p$ & n.s. & n.s. & - & - \\
\hline \multirow{3}{*}{$\begin{array}{l}\text { 3-year overall } \\
\text { survival }\end{array}$} & Open & $90.4 \%$ & $83.6 \%$ & - & - \\
\hline & Laparoscopic & $91.7 \%$ & $86.7 \%$ & - & - \\
\hline & $p$ & n.s. & n.s. & - & - \\
\hline
\end{tabular}

trials of laparoscopic vs open approaches for TME 
*pCRM (Pathological circumferential resection margin); ** TME (Total mesorectal excision); n.s. (Not significant) 